\title{
A Novel Approach for Finding a Shortest Path in a Mixed Fuzzy Network
}

\author{
Ali Tajdin ${ }^{1}$, Iraj Mahdavi ${ }^{1 *}$, Nezam Mahdavi-Amiri ${ }^{2}$, Bahram Sadeghpour-Gildeh $^{3}$, \\ Reza Hassanzadeh ${ }^{1}$ \\ ${ }^{I}$ Department of Industrial Engineering, Mazandaran University of Science and Technology, Babol, Iran \\ ${ }^{2}$ Faculty of Mathematical Sciences, Sharif University of Technology, Tehran, Iran \\ ${ }^{3}$ Department of Mathematics and Statistics, Mazandaran University, Babolsar, Iran \\ E-mail:irajarash@rediffmail.com \\ Received October 11, 2009; revised November 13, 2009; accepted November 14, 2009
}

\begin{abstract}
We present a novel approach for computing a shortest path in a mixed fuzzy network, network having various fuzzy arc lengths. First, we develop a new technique for the addition of various fuzzy numbers in a path using $\alpha$-cuts. Then, we present a dynamic programming method for finding a shortest path in the network. For this, we apply a recently proposed distance function for comparison of fuzzy numbers. Four examples are worked out to illustrate the applicability of the proposed approach as compared to two other methods in the literature as well as demonstrate the novel feature offered by our algorithm to find a fuzzy shortest path in mixed fuzzy networks with various settings for the fuzzy arc lengths.
\end{abstract}

Keywords: Fuzzy Numbers, $\alpha$-Cut; Shortest Path, Dynamic Programming

\section{Introduction}

Determination of shortest distance and shortest path between two vertices is one of the most fundamental problems in graph theory. Let $G=(V, E)$ be a graph with $V$ as the set of vertices and $E$ as the set of edges. A path between two vertices is an alternating sequence of vertices and edges starting and ending with vertices, and no vertices or no edges appear more than once in the sequence. The length of a path is the sum of the weights of the edges on the path. There may exist more than one path between a pair of vertices. The shortest path problem is to find the path with minimum length between a specified pair of vertices. In classical graph theory, the weight of each edge is taken as a crisp real number. But, practically weight of each edge of the network may not be a fixed real number and it may well be imprecise.

The shortest path problem involves addition and comparison of the edge weights. Since, the addition and comparison between two interval numbers or between two triangular fuzzy numbers are not alike those between two precise real numbers, it is necessary to discuss them at first. Interval arithmetic was developed in Moore [1]. The case of optimization with interval valued and fuzzy constraints were discussed in Delgado et al., Ishibuchi and Tanaka, Sengupta, and Shaocheng [2-5]. Various ranking methods for interval numbers were introduced by several researchers. An extensive survey of the order relations along with a new proposal are given by Sengupta and Pal [6]. There are also ranking methods for fuzzy numbers available in the literature. Dubois and Prade [7] introduced a ranking of fuzzy numbers in the setting of possibility theory, and Chen [8] ranked fuzzy numbers using maximizing and minimizing sets. Ranking of fuzzy numbers was also studied by Bortolan and Degani, Cheng, and Delgado et al. [2,9,10].

Fuzzy graph problems were addressed in Blue et al. and Koczy, Klein, Li et al., Lin and Chen, Okada and Gen [11-17] paid special attention to fuzzy shortest paths. In a recent development, Okada and Soper [18] proposed an algorithm to find the shortest path in a network with fuzzy edge weights. The algorithm gives a family of non-dominated shortest paths for a specified satisfaction level, but it does provide any guideline to the decisionmaker to choose the best amongst the paths according to his/her view; i.e., optimistic, pessimistic, etc.

The shortest path (SP) problem has received lots of attention from researchers in the past decades, because it is important to many applications such as communication, transportation, scheduling and routing. In a network, the 
arc length may represent time or cost. Conventionally, it is assumed to be crisp. However, it is difficult for decision makers to specify the arc lengths. For example, using the same modem to transmit the data from node $a$ to $b$ in a network, the data transmission time may not be the same every time. Therefore, in real world, the arc length could be uncertain. Fuzzy set theory, as proposed by Zadeh [19], is frequently utilized to deal with uncertainty. Zadeh presented the possibility theory using membership functions to describe uncertainties.

Considering a directed network that is composed of a finite set of nodes and a set of directed arcs, we denote each arc by an ordered pair $(i, j)$, where $i$ and $j$ are two different nodes. The arc length represents the distance needed to traverse $(i, j)$ from node $i$ to $j$. We denote it by $l(i, j)$ or $L(i, j)$, when it is crisp or fuzzy, respectively. We call $L(i, j)$ as "fuzzy arc length".

The shortest path problem is the following: given a weighted, directed graph and two special vertices $s$ and $t$, compute the weight of the shortest path between $s$ and $t$. The shortest path problem is one of the most fundamental network optimization problems. This problem comes up in practice and arises as a subproblem in many network optimization algorithms. Algorithms for this problem have been studied for a long time [20-22]. However, advances in the method and theory of shortest path algorithms are still being made [23-25].

In the network we consider here, the lengths of the arcs are assumed to represent transportation times or costs rather than geographical distances. As time or cost fluctuate with traffic conditions, payload and so on, it is not practical to represent the arcs as crisp values. Thus, it is appropriate to utilize fuzzy numbers based on fuzzy set theory. In proposing an algorithm for solving the problem, we are first faced with the comparison or ordering of fuzzy numbers, a task not considered to be routine. For this reason, fuzzy shortest path problems have rarely been studied despite their potential application to many problems $[18,26]$. The problem turns out to be even more complicated in our more general case of allowing various fuzzy arc lengths.

Here, we propose a new approach and an algorithm to find a shortest path in a mixed network having various fuzzy arc lengths. The remainder of the paper is organized as follows. In Section 2, basic concepts and definitions are given. A dynamic programming algorithm for finding a fuzzy shortest path in a network is presented in Section 3. There, we make use of $\alpha$-cuts for computing approximations for the addition of two different types of fuzzy numbers and apply a distance function for the comparison of fuzzy numbers. Comparative examples are given in Section 4. Section 5 works out an example to show the novel feature of our algorithm to find fuzzy shortest paths in mixed fuzzy networks with various settings for the fuzzy arc lengths. We conclude in Section 6.

\section{Concepts and Definitions}

We start with basic definitions of some well-known fuzzy numbers.

Definition 1. An $L R$ fuzzy number is represented by $\tilde{A}=(m, \alpha, \beta)_{L R}$, with the membership function, $\mu_{\tilde{a}}(x)$, defined by the expression,

$$
\mu_{\tilde{a}}(x)= \begin{cases}L\left[\frac{m-x}{\alpha}\right] & x \leq m \\ R\left[\frac{x-m}{\beta}\right] & x \geq m,\end{cases}
$$

where $L$ and $R$ are non-increasing functions from $R^{+}$to $[0,1], L(0)=R(0)=1, m$ is the center, $\alpha$ is the left spread and $\beta$ is the right spread.

Note that if $L(x)=R(x)=1-x$ with $0<x<1$, then $x$ is a triangular fuzzy number and is represented by the triplet $\tilde{a}=\left(a_{1}, a_{2}, a_{3}\right)$, with the membership function, $\mu_{\tilde{a}}(x)$, defined by

$$
\mu_{\tilde{a}}(x)=\left\{\begin{array}{cc}
0 & x \leq a_{1} \\
\frac{x-a_{1}}{a_{2}-a_{1}} & a_{1}<x \leq a_{2} \\
\frac{a_{3}-x}{a_{3}-a_{2}} & a_{2}<x \leq a_{3} \\
0 & x>a_{3} .
\end{array}\right.
$$

A triangular fuzzy number is shown in Figure 1.

Definition 2. A trapezoidal fuzzy number $\tilde{a}$ is shown by $\tilde{a}=\left(a_{1}, a_{2}, a_{3}, a_{4}\right)$, with the membership function as follows:

$$
\mu_{\tilde{a}}(x)=\left\{\begin{array}{cc}
0 & x \leq a_{1} \\
\frac{x-a_{1}}{a_{2}-a_{1}} & a_{1} \leq x \leq a_{2} \\
1 & a_{2} \leq x \leq a_{3} \\
\frac{a_{4}-x}{a_{4}-a_{3}} & a_{3} \leq x \leq a_{4} \\
0 & a_{4} \leq x .
\end{array}\right.
$$

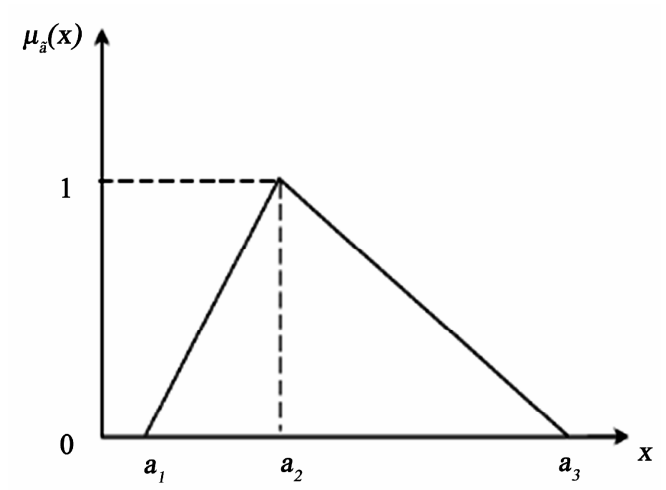

Figure 1. A triangular fuzzy number. 
A general trapezoidal fuzzy number is shown in Figure 2. It is apparent that a triangular fuzzy number is a special trapezoidal fuzzy number with $a_{2}=a_{3}$.

Definition 3. If $L(x)=R(x)=e^{-x^{2}}$, with $x \in \mathfrak{R}$, then $x$ is a normal fuzzy number that is shown by $(m, \sigma)$ and the corresponding membership function is defined to be:

$$
\mu_{\tilde{a}}(x)=e^{-\left(\frac{x-m}{\sigma}\right)^{2}}, x \in \Re,
$$

where $m$ is the mean and $\sigma$ is the standard deviation. A normal fuzzy number is shown in Figure 3.

Definition 4. The $\alpha$-cut and strong $\alpha$-cut for a fuzzy set $\tilde{A}$ are shown by $\tilde{A}_{\alpha}$ and $\tilde{A}_{\alpha}^{+}$, respectively, and for $\alpha \in[0,1]$ are defined to be:

$$
\begin{aligned}
& \tilde{A}_{\alpha}=\left\{x \mid \mu_{\tilde{A}}(x) \geq \alpha, x \in X\right\}, \\
& \tilde{A}_{\alpha}^{+}=\left\{x \mid \mu_{\tilde{A}}(x)>\alpha, x \in X\right\},
\end{aligned}
$$

where $X$ is the universal set.

Upper and lower bounds for any $\alpha$-cut $\left(\tilde{A}_{\alpha}\right)$ are shown by $\sup \tilde{A}_{\alpha}$ and $\inf \tilde{A}_{\alpha}$, respectively. Here, we assume that the upper and lower bounds of $\alpha$-cuts are

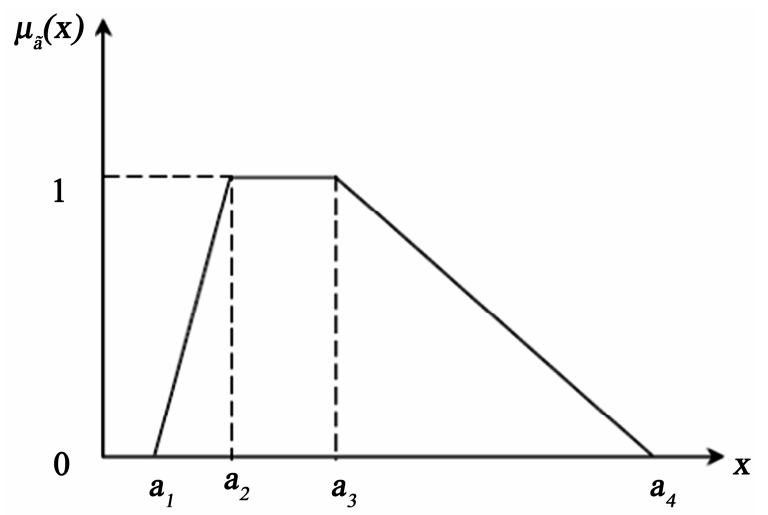

Figure 2. A trapezoidal fuzzy number.

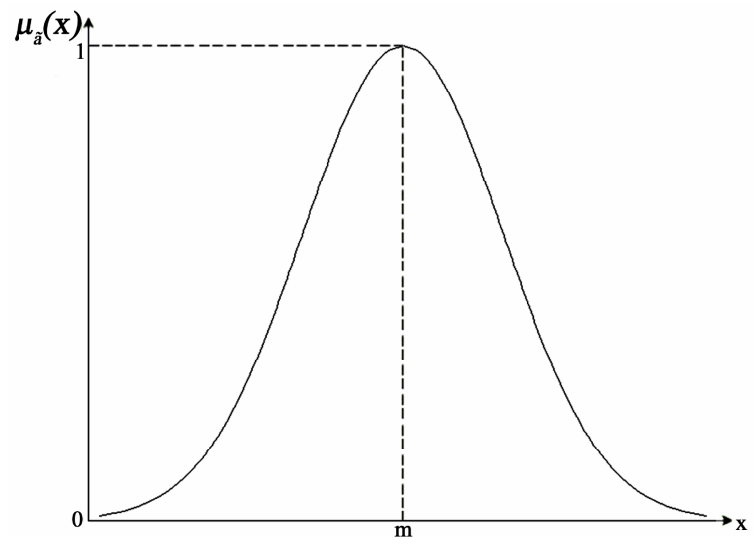

Figure 3. A normal fuzzy number. finite values and for simplicity we show $\sup \tilde{A}_{\alpha}$ by $A_{\alpha}^{+}$and $\inf \tilde{A}_{\alpha}$ by $A_{\alpha}^{-}$.

\subsection{Computing $\alpha$-Cuts for Fuzzy Numbers}

For an $L R$ fuzzy number with $L$ and $R$ as invertible functions, the $\alpha$-cuts are:

$$
\begin{aligned}
& \alpha=L\left[\frac{m-x}{\beta}\right] \Rightarrow \frac{m-x}{\beta}=L^{-1}(\alpha) \Rightarrow x=m-\beta L^{-1}(\alpha), \\
& \alpha=R\left[\frac{x-m}{\gamma}\right] \Rightarrow \frac{x-m}{\gamma}=R^{-1}(\alpha) \Rightarrow x=m+\gamma R^{-1}(\alpha) .
\end{aligned}
$$

For specific $L$ and $R$ functions, the following cases are discussed. Let $\tilde{a}=\left(a_{1}, a_{2}, a_{3}, a_{4}\right)$ be a trapezoidal fuzzy number. The $\alpha$-cut for $\tilde{a},\left(\tilde{a}_{\alpha}\right)$, is computed as:

$$
\begin{aligned}
\mu_{20}(x) & =\left\{\begin{array}{cc}
0 & x \leq a_{1} \\
\frac{x-a_{1}}{a_{2}-a_{1}} & a_{1} \leq x \leq a_{2} \\
1 & a_{2} \leq x \leq a_{3} \\
\frac{a_{4}-x}{a_{4}-a_{3}} & a_{3} \leq x \leq a_{4} \\
0 & a_{4} \leq x
\end{array}\right. \\
\Rightarrow \quad \alpha & =\frac{x-a_{1}}{a_{2}-a_{1}} \Rightarrow \quad x=\left(a_{2}-a_{1}\right) \alpha+a_{1} \\
\Rightarrow \quad & \frac{a_{4}-x}{a_{4}-a_{3}} \Rightarrow \quad x=a_{4}-\left(a_{4}-a_{3}\right) \alpha \\
\tilde{a}_{\alpha} & = \begin{cases}\tilde{a}_{\alpha}^{+}=a_{4}-\left(a_{4}-a_{3}\right) \alpha \quad, \quad 0 \leq \alpha \leq 1 . \\
\tilde{a}_{\alpha}^{-}=\left(a_{2}-a_{1}\right) \alpha+a_{1}\end{cases}
\end{aligned}
$$

Note that the $\alpha$-cut for triangular fuzzy numbers is simply obtained by using the above equations considering $a_{2}=a_{3}$ :

$$
\tilde{a}_{\alpha}=\left\{\begin{array}{l}
\tilde{a}_{\alpha}^{+}=a_{3}-\left(a_{3}-a_{2}\right) \alpha \\
\tilde{a}_{\alpha}^{-}=\left(a_{2}-a_{1}\right) \alpha_{\alpha}+a_{1}
\end{array} \quad, \quad 0 \leq \alpha \leq 1 .\right.
$$

For $\tilde{a}=(m, \sigma)$, a normal fuzzy number, $\tilde{a}_{\alpha}$, is computed as:

$$
\begin{aligned}
& \alpha=e^{-\left(\frac{m-x}{\sigma}\right)_{2}} \Rightarrow \sqrt{-\ln (\alpha)}=\frac{m-x}{\sigma} \\
& \alpha=e^{-\left(\frac{x-m}{\sigma}\right)_{2}} \Rightarrow \sqrt{-\ln (\alpha)}=\frac{x-m}{\sigma} \\
& \Rightarrow \tilde{a}_{\alpha}=\left\{\begin{array}{l}
\tilde{a}_{\alpha}^{L}=m-\sigma \sqrt{-\ln \alpha} \\
\tilde{a}_{\alpha}^{R}=m+\sigma \sqrt{-\ln \alpha}
\end{array} \quad, \quad 0<\alpha \leq 1 .\right.
\end{aligned}
$$




\subsection{Fuzzy Sum Operators}

Here, we propose an approach for summing various fuzzy numbers approximately using $\alpha$-cuts. The approximation is based on fitting an appropriate model for the sum using $\alpha$-cuts of the addition by a set of $\alpha_{i}$ values. Let us divide the $\alpha$-interval $[0,1]$ into $n$ equal subintervals by letting $\alpha_{0}=0, \alpha_{i}=\alpha_{i-1}+\Delta \alpha_{i}$, $i=1, \ldots, n$ with $\Delta \alpha_{i}=\frac{1}{n}, i=1, \ldots, n$. This way, we have a set of $n+1$ equidistant $\alpha_{i}$ points. For addition of two different fuzzy numbers, we add the set of corresponding $\alpha_{i}$-cut points of the two numbers to yield the $\alpha_{i}$-cuts of the sum as an approximation for the fuzzy addition.

\section{An Algorithm for Fuzzy Shortest Path in a Network}

\subsection{Distance between Fuzzy Numbers}

Knowing that we can obtain a good approximation of the addition of various fuzzy numbers by use of $\alpha$-cuts, we compute the distance between two fuzzy numbers using the resulting points from the $\alpha$-cuts. For $\tilde{a}$ and $\tilde{b}$ as two different fuzzy numbers, we use a new fuzzy ranking method for the fuzzy numbers. Let us consider the fuzzy min operation to be defined as follows

$$
\begin{aligned}
M V^{\mathscr{O}} & =\operatorname{Min} \quad \text { value }(d, d) \rho \\
& =\left(\min \left(a_{1}, b_{1}\right), \min \left(a_{2}, b_{2}\right), \min \left(a_{3}, b_{3}\right), \min \left(a_{4}, b_{4}\right)\right) .
\end{aligned}
$$

It is evident that, for non-comparable fuzzy numbers $\tilde{a}$ and $\tilde{b}$, the fuzzy $\min$ operation results in a fuzzy number different from both of them. For example, for $\tilde{a}=(5,10,13,19)$ and $\tilde{b}=(6,9,15,20)$, we get from (4) a fuzzy $M \tilde{V}=(5,9,13,19)$ which is different from both $\tilde{a}$ and $\tilde{b}$. To alleviate this drawback, we use a method based on the distance between fuzzy numbers. We use the distance function introduced by Sadeghpour-Gildeh and Gien [27]. The main advantages of this distance function are the generality of its usage on various fuzzy numbers, and its reliability in distinguishing unequal fuzzy numbers. Indeed, the use of this distance function worked out to be quite appropriate for our approach here as well as in a different context before where we considered the arc lengths in the network to be all of the same type (see Mahdavi et al. [28]).

The proposed $D_{p, q}$-distance, indexed by parameters $1<p<\infty$ and $0<q<1$, between two fuzzy numbers $\mathscr{a}$ and $b^{\prime}$ is a nonnegative function given by:

The analytical properties of $D_{p, q}$ depend on the first

$$
D_{p, q}(\&, \phi)= \begin{cases}{\left[(1-q) \int_{0}^{1}\left|a_{\alpha}^{-}-b_{\alpha}^{-}\right|^{p} d \alpha+q \int_{0}^{1}\left|a_{\alpha}^{+}-b_{\alpha}^{+}\right|^{p} d \alpha\right]^{\frac{1}{p}},} & p<\infty, \\ (1-q) \sup _{0<\alpha \leq 1}\left(\left|a_{\alpha}^{-}-b_{\alpha}^{-}\right|\right)+q \inf _{0<\alpha \leq 1}\left(\left|a_{\alpha}^{+}-b_{\alpha}^{+}\right|\right), & p=\infty .\end{cases}
$$

parameter $p$, while the second parameter $q$ of $D_{p, q}$ characterizes the subjective weight attributed to the end points of the support; i.e., $\left(a_{\alpha}^{+}, a_{\alpha}^{-}\right)$of the fuzzy numbers. If there is no reason for distinguishing any side of the fuzzy numbers, then $D_{p, \frac{1}{2}}$ is recommended.

Having $q$ close to 1 results in considering the right side of the support of the fuzzy numbers more favorably. Since the significance of the end points of the support of the fuzzy numbers is assumed to be the same, then we consider $q=\frac{1}{2}$.

For two fuzzy numbers $\tilde{a}$ and $\tilde{b}$ with corresponding $\alpha_{i}$-cuts, the $D_{p, q}$ distance is approximately proportional to:

$$
D_{p, q}(\tilde{a}, \tilde{b})=\left[(1-q) \sum_{i=1}^{n}\left|a_{\alpha_{i}}^{-}-b_{\alpha_{i}}^{-}\right|^{p}+q \sum_{i=1}^{n}\left|a_{\alpha_{i}}^{+}-b_{\alpha_{i}}^{+}\right|^{p}\right]^{\frac{1}{p}} .
$$

If $q=\frac{1}{2}, p=2$, then the above equation turns into:

$$
D_{2, \frac{1}{2}}(\tilde{a}, \tilde{b})=\sqrt{\frac{1}{2} \sum_{i=1}^{n}\left(a_{\alpha_{i}}^{-}-b_{\alpha_{i}}^{-}\right)^{2}+\frac{1}{2} \sum_{i=1}^{n}\left(a_{\alpha_{i}}^{+}-b_{\alpha_{i}}^{+}\right)^{2}},
$$

To compare two fuzzy arc lengths $\tilde{a}$ and $\tilde{b}$ with $\alpha_{i}$-cuts as their approximations, since they are supposed to represent positive values, we compare them with $M \tilde{V}=(0,0, \ldots, 0)$. In fact, we use (7) to compute $D_{2, \frac{1}{2}}(\tilde{a}, 0)$ and $D_{2, \frac{1}{2}}(\tilde{b}, 0)$ and use these values for comparison of the two numbers. 


\subsection{An Algorithm for Computing s Shortest Fuzzy Path}

The following dynamic programming algorithm is for computing the shortest path in a network. The algorithm is based on Floyd's dynamic programming method to find a shortest path, if it exists, between every pair of nodes $i$ and $j$ in the network (see Floyd [29]).

We make use of the following optimal value function $f_{k}(i, j)$ and the corresponding labeling function $P_{k}(i, j)$ : $f_{k}(i, j)=$ length of the shortest path from node $i$ to node $j$ when the path is considered to use only the nodes from the set of nodes $\{1, \ldots, k\}$.

$P_{k}(i, j)=$ the last intermediate node on the shortest path from node $i$ to node $j$ using $\{1, \ldots ., k\}$ as intermediate node.

The dynamic updating for the optimal path length and its corresponding labeling are:

$$
f_{k}(i, j)=\min \left\{f_{k-1}(i, j), f_{k-1}(i, k)+f_{k-1}(k, j)\right\},
$$

$$
P_{k}(i, j)= \begin{cases}P_{k-1}(i, j) & \text { if } \mathrm{k} \text { is not on shortest path from } \mathrm{i} \text { to } \mathrm{j} \text { using }\{1, \ldots, \mathrm{k}\} \\ P_{k-1}(k, j) & \text { otherwise. }\end{cases}
$$

We are now ready to give the steps of the algorithm.

Algorithm: A dynamic programming method for computing a shortest path in a fuzzy network $G=(V, A)$, where $V$ is the set of nodes with $|V|=N$, and $A$ is the set of arcs.

Step 1: Let $k=0$ and $\tilde{f}_{k}(i, j)=\tilde{d}_{i j}$, for all $(i, j) \in A$, $\tilde{f}_{k}=(i, j)=\infty$, for all $(i, j) \notin A$. If an arc exists from node $i$ to node $j$ then let $P_{k}(i, j)=i$.

Step 2: Let $k=k+1$.

Do the following steps for $i=1,2,3, \ldots ., N, j=1,2,3, \ldots ., N, i \neq j$.

2.1 Compute the value of $f_{k}(i, j)=\min$ $\left[f_{k-1}(i, j), f_{k-1}(i, k)+f_{k-1}(k, j)\right]$ (for the addition, our proposed method discussed in Subection 2.2 and for comparison of fuzzy numbers the $D_{p, q}$ method of Subection 3.1 are applied).

2.2 If node $k$ is not on the shortest path using nodes $\{1,2, \ldots, k\}$ as intermediate nodes, then let $P_{k}(i, j)=$ $P_{k-1}(i, j)$ else let $P_{k}(i, j)=P_{k-1}(k, j)$

\section{Step 3: If $k<N$ then go to Step 2.}

Step 4: Obtain the shortest path using the $P_{k}(i, j)$. If $f_{N}(i, j)=\infty$, then there is no path between $i$ and $j$. The shortest path from node $i$ to $j$, if it exists, is identified backwards and read by the nodes: $j, P_{N}(i, j)=k$ followed by $P_{N}(i, k), \ldots, P_{N}(i, l)=i$, where $l$ is the node immediately after $i$ in the path.

\subsection{Termination and Complexity of the Algorithm}

The proposed algorithm terminates after $N$ outer iterations corresponding to $k$. A total of $N(N-1)^{2}$ additions and comparisons are needed for every $k$. For each addition, $n$ fuzzy additions for the $\alpha_{i}$-cuts should be performed resulting in $2 n(N)(N-1)^{2}$ additions. For comparisons, we have $(2 n+1) N(N-1)^{2}$ additions and $(2 n+1) N(N-1)^{2}$ multiplications using (7). Therefore, the total needed operations are $(6 n+2) N(N-1)^{2}$ additions and multiplications, with $N(N-1)^{2}$ comparison

\section{Comparative Examples}

Here, we illustrate examples for a comparison of our proposed method and two other approaches.

\section{Example 1:}

Consider the following network Figure 4 considered by Chuang and Kung [30]. The triangular arc lengths are presented in Table 1. The results obtained by the approach $\left(\tilde{f}_{6}\right.$ and $\left.P_{6}\right)$ of Chuang and Kung [30] are shown in Tables 2 and 3.

The shortest path and the corresponding length using the proposed approach in Chuang and Kung [30] are reported below:

Shortest path from 1 to $6: 1 \rightarrow 2 \rightarrow 4 \rightarrow 6$.

Shortest path length from 1 to $6:(177,195,256)$

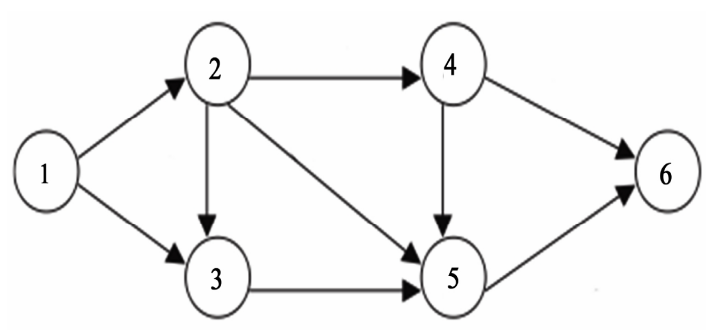

Figure 4. The network for Example 1.

Table 1. The arc lengths for example 1.

\begin{tabular}{cccccc}
\hline Arc & lengths & Arc & lengths & Arc & lengths \\
\hline$(1,2)$ & $(33,45,50)$ & $(1,3)$ & $(42,57,61)$ & $(2,3)$ & $(50,52,61)$ \\
$(2,4)$ & $(56,58,72)$ & $(2,5)$ & $(51,79,85)$ & $(3,5)$ & $(43,55,60)$ \\
$(4,5)$ & $(32,40,46)$ & $(4,6)$ & $(88,92,134)$ & $(5,6)$ & $(75,110,114)$ \\
\hline
\end{tabular}


Table 2. The $\tilde{f}_{6}$ values obtained by the approach of Chuang and Kung.

\begin{tabular}{ccccccc}
\hline $\boldsymbol{i} / \boldsymbol{j}$ & $\mathbf{1}$ & $\mathbf{2}$ & $\mathbf{3}$ & $\mathbf{4}$ & $\mathbf{5}$ & $\mathbf{6}$ \\
\hline $\mathbf{1}$ & - & $(33,45,50)$ & $(42,57,61)$ & $(89,103,122)$ & $(85,112,121)$ & $(177,195,256)$ \\
$\mathbf{2}$ & - & - & $(50,52,61)$ & $(56,58,72)$ & $(51,79,85)$ & $(144,150,206)$ \\
$\mathbf{3}$ & - & - & - & - & $(43,55,60)$ & $(118,165,174)$ \\
$\mathbf{4}$ & - & - & - & - & $(32,40,46)$ & $(88,92,134)$ \\
$\mathbf{5}$ & - & - & - & - & - & $(75,110,114)$ \\
$\mathbf{6}$ & - & - & - & - & - & - \\
\hline
\end{tabular}

Table 3. The $P_{6}$ values obtained by the approach of Chuang and Kung.

\begin{tabular}{lllllll}
\hline $\boldsymbol{i} / \boldsymbol{j}$ & $\mathbf{1}$ & $\mathbf{2}$ & $\mathbf{3}$ & $\mathbf{4}$ & $\mathbf{5}$ & $\mathbf{6}$ \\
\hline $\mathbf{1}$ & - & 1 & 1 & 2 & 3 & 4 \\
$\mathbf{2}$ & - & - & 2 & 2 & 2 & 4 \\
$\mathbf{3}$ & - & - & - & - & 3 & 5 \\
$\mathbf{4}$ & - & - & - & - & 4 & 4 \\
$\mathbf{5}$ & - & - & - & - & - & 5 \\
$\mathbf{6}$ & - & - & - & - & - & - \\
\hline
\end{tabular}

Here, we solve the same problem using our proposed algorithm givenin Subection 3.2 using the ranking method of Sadeghpour-Gildeh and Gien [27]. The results of the proposed approach for $\tilde{f}_{6}$ and $P_{6}$ are given in Tables 4 and 5.

Here, the shortest path obtained and the corresponding length are exactly the same as the ones we obtained by the approach of Chuang and Kung [30].

Example 2:

Consider the following network Figure 5 considered

Table 4. The $\tilde{f}_{6}$ values obtained by our proposed algorithm

\begin{tabular}{ccccccc}
\hline $\boldsymbol{i} / \boldsymbol{j}$ & $\mathbf{1}$ & $\mathbf{2}$ & $\mathbf{3}$ & $\mathbf{4}$ & $\mathbf{5}$ & $\mathbf{6}$ \\
\hline $\mathbf{1}$ & - & $(33,45,50)$ & $(42,57,61)$ & $(89,103,122)$ & $(85,112,121)$ & $(177,195,256)$ \\
$\mathbf{2}$ & - & - & $(50,52,61)$ & $(56,58,72)$ & $(51,79,85)$ & $(144,150,206)$ \\
$\mathbf{3}$ & - & - & - & - & $(43,55,60)$ & $(118,165,174)$ \\
$\mathbf{4}$ & - & - & - & - & $(32,40,46)$ & $(88,92,134)$ \\
$\mathbf{5}$ & - & - & - & - & - & $(75,110,114)$ \\
$\mathbf{6}$ & - & - & - & - & - & - \\
\hline
\end{tabular}

by Hernandes et al. [32]. The fuzzy triangular arc lengths are given in Table 6 . The results $\left(\tilde{f}_{6}\right.$ and $\left.P_{6}\right)$ for the approach of Hernandes et al. [32] are given in Tables 7 and 8.

The shortest path and the corresponding length using the proposed approach of Hernandes et al. [32] are reported below:

Shortest path from 1 to $11: 1 \rightarrow 9 \rightarrow 7 \rightarrow 11$.

Shortest path length from 1 to $11:(860,902,990)$

We solved the same problem using our proposed algorithm of Subsection 3.2 using the ranking method of Sadeghpour-Gildeh and Gien [27]. The results of our proposed approach $\left(\tilde{f}_{11}\right.$ and $\left.P_{11}\right)$ are given in Tables 9 and 10.

The shortest path and the corresponding length are reported below:

Shortest path from 1 to $11: 1 \rightarrow 9 \rightarrow 7 \rightarrow 11$.

Shortest path length from 1 to $11:(840,882,990)$.

Clearly, the proposed algorithm computes almost the same solution as obtained by Hernandes et al. [32].
Table 5. The $P_{6}$ values obtained by our proposed algorithm.

\begin{tabular}{ccccccc}
\hline $\boldsymbol{i} / \boldsymbol{j}$ & $\mathbf{1}$ & $\mathbf{2}$ & $\mathbf{3}$ & $\mathbf{4}$ & $\mathbf{5}$ & $\mathbf{6}$ \\
\hline $\mathbf{1}$ & - & 1 & 1 & 2 & 3 & 4 \\
$\mathbf{2}$ & - & - & 2 & 2 & 2 & 4 \\
$\mathbf{3}$ & - & - & - & - & 3 & 5 \\
$\mathbf{4}$ & - & - & - & - & 4 & 4 \\
$\mathbf{5}$ & - & - & - & - & - & 5 \\
$\mathbf{6}$ & - & - & - & - & - & - \\
\hline
\end{tabular}

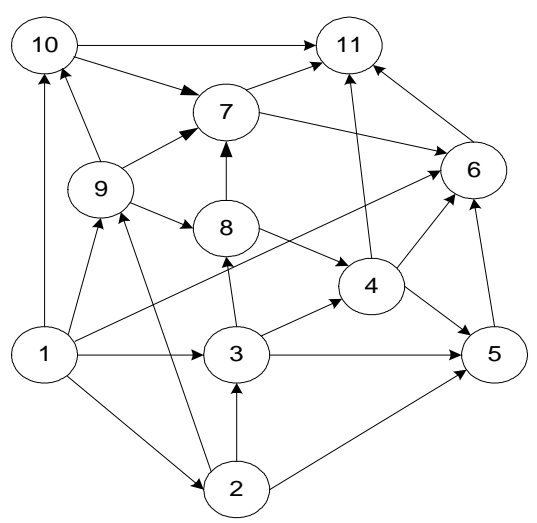

Figure 5. The network for Example 2. 
Table 6. The arc lengths for Example 2.

\begin{tabular}{cccccc}
\hline Arc & lengths & Arc & lengths & Arc & lengths \\
\hline$(1,2)$ & $(800,820,840)$ & $(3,5)$ & $(730,748,770)$ & $(8,4)$ & $(710,730,735)$ \\
$(1,3)$ & $(350,361,370)$ & $(3,8)$ & $(425,443,465)$ & $(8,7)$ & $(230,242,255)$ \\
$(1,6)$ & $(650,677,683)$ & $(4,5)$ & $(190,199,210)$ & $(9,7)$ & $(120,130,150)$ \\
$(1,9)$ & $(290,300,350)$ & $(4,6)$ & $(310,340,360)$ & $(9,8)$ & $(130,137,145)$ \\
$(1,10)$ & $(420,450,470)$ & $(4,11)$ & $(710,740,770)$ & $(9,10)$ & $(230,242,260)$ \\
$(2,3)$ & $(180,186,193)$ & $(5,6)$ & $(610,660,690)$ & $(10,7)$ & $(330,342,350)$ \\
$(2,5)$ & $(495,510,525)$ & $(6,11)$ & $(230,242,260)$ & $(10,11)$ & $(1250,1310,1440)$ \\
$(2,9)$ & $(900,930,960)$ & $(7,6)$ & $(390,410,440)$ & $(3,4)$ & $(650,667,883)$ \\
$(7,11)$ & $(450,472,490)$ & & & & \\
\hline
\end{tabular}

Table 7. The $\tilde{f}_{11}(i, j)$ values obtained by Hernandes et al.

\begin{tabular}{cccccccc}
\hline $\boldsymbol{i} / \boldsymbol{j}$ & $\mathbf{1}$ & $\mathbf{2}$ & $\mathbf{3}$ & $\mathbf{4}$ & $\mathbf{5}$ & $\mathbf{6}$ & $\mathbf{7}$ \\
\hline $\mathbf{1}$ & - & $(800,820,840)$ & $(350,361,370)$ & $(1000,1028,1253)$ & $(1080,1109,1140)$ & $(650,677,683)$ & $(410,430,500)$ \\
$\mathbf{2}$ & - & - & $(180,186,193)$ & $(830,853,1076)$ & $(495,510,525)$ & $(1105,1170,1215)$ & $(835,871,913)$ \\
$\mathbf{3}$ & - & - & - & $(650,667,883)$ & $(730,748,770)$ & $(960,1007,1243)$ & $(655,685,720)$ \\
$\mathbf{4}$ & - & - & - & - & $(190,199,210)$ & $(310,340,360)$ & - \\
$\mathbf{5}$ & - & - & - & - & - & $(610,660,690)$ & - \\
$\mathbf{6}$ & - & - & - & - & - & - & - \\
$\mathbf{7}$ & - & - & - & - & - & $(390,410,440)$ & - \\
$\mathbf{8}$ & - & - & - & $(710,730,735)$ & $(900,929,945)$ & $(620,652,695)$ & $(230,242,255)$ \\
$\mathbf{9}$ & - & - & - & $(840,867,880)$ & $(1030,1066,1090)$ & $(510,540,590)$ & $(120,130,150)$ \\
$\mathbf{1 0}$ & - & - & - & - & - & $(720,752,790)$ & $(330,342,350)$ \\
$\mathbf{1 1}$ & - & - & - & - & - & - & - \\
\hline
\end{tabular}

Table 7. continued.

\begin{tabular}{|c|c|c|c|c|}
\hline$i / j$ & 8 & 9 & 10 & 11 \\
\hline 1 & $(420,437,495)$ & $(290,300,350)$ & $(420,450,470)$ & $(860,902,990)$ \\
\hline 2 & $(605,629,658)$ & $(900,930,960)$ & $(1130,1172,1220)$ & $(1285,1343,1403)$ \\
\hline 3 & $(425,443,465)$ & - & - & $(1105,1157,1210)$ \\
\hline 4 & - & - & - & $(540,582,620)$ \\
\hline 5 & - & - & - & $(840,902,950)$ \\
\hline 6 & - & - & - & $(230,242,260)$ \\
\hline 7 & - & - & - & $(450,472,490)$ \\
\hline 8 & - & - & - & $(680,714,745)$ \\
\hline 9 & $(130,137,145)$ & - & $(230,242,260)$ & $(570,602,640)$ \\
\hline 10 & - & - & - & $(780,814,840)$ \\
\hline 11 & - & - & - & - \\
\hline
\end{tabular}

\section{Example 3: A wireless sensor network}

Consider a mobile service company which handles 23 geographical centers. A configuration of a telecommunication network is presented in Figure 6. Assume that the distance between any two centers is a trapezoidal fuzzy number (the arc lengths are given in Table 11). The company wants to find a shortest path for an effective message flow amongst the centers.

The results obtained by our approach $\left(\tilde{f}_{23}\right.$ and $\left.P_{23}\right)$ are given in Tables 12 and 13.

The shortest path and the corresponding length are reported below:

Shortest path from 1 to $23: 1 \rightarrow 5 \rightarrow 11 \rightarrow 17 \rightarrow 21 \rightarrow 23$. 
Shortest path length from 1 to $23:(38,49,58,65)$.

\section{Discussion}

We can also apply the proposed algorithm to networks having possibly a mixture of different fuzzy numbers as arc lengths. To see how the steps of the proposed algorithm are carried out on such networks, a small sized mixed fuzzy network with 4 nodes as shown in Figure 7 is considered, where the arc lengths are considered to be a mixture of trapezoidal and normal fuzzy numbers.

Example 4: Consider the mixed fuzzy network in Figure 4 with four nodes and five arcs having two trapezoidal and three normal arc lengths as specified in Table 14.

Step 1: We gain the $\tilde{f}_{k}(i, j)=\tilde{d}_{i j}$ for $k=0$ as specified in Table 14.

Table 8. The $P_{11}(i, j)$ values obtained by Hernandes et al.

\begin{tabular}{cccccccccccc}
\hline $\boldsymbol{i} / \boldsymbol{j}$ & $\mathbf{1}$ & $\mathbf{2}$ & $\mathbf{3}$ & $\mathbf{4}$ & $\mathbf{5}$ & $\mathbf{6}$ & $\mathbf{7}$ & $\mathbf{8}$ & $\mathbf{9}$ & $\mathbf{1 0}$ & $\mathbf{1 1}$ \\
\hline $\mathbf{1}$ & - & 1 & 1 & 3 & 3 & 1 & 9 & 9 & 1 & 1 & 9 \\
$\mathbf{2}$ & - & - & 2 & 3 & 2 & 5 & 8 & 3 & 2 & 9 & 8 \\
$\mathbf{3}$ & - & - & - & 3 & 3 & 4 & 8 & 3 & - & - & 8 \\
$\mathbf{4}$ & - & - & - & - & 4 & 4 & - & - & - & - & 6 \\
$\mathbf{5}$ & - & - & - & - & - & 5 & - & - & - & - & 6 \\
$\mathbf{6}$ & - & - & - & - & - & - & - & - & - & - & 6 \\
$\mathbf{7}$ & - & - & - & - & - & 7 & - & - & - & - & 7 \\
$\mathbf{8}$ & - & - & - & 8 & 4 & 7 & 8 & - & - & - & 7 \\
$\mathbf{9}$ & - & - & - & 8 & 8 & 7 & 9 & 9 & - & 9 & 7 \\
$\mathbf{1 0}$ & - & - & - & - & - & 7 & 10 & - & - & - & 7 \\
$\mathbf{1 1}$ & - & - & - & - & - & - & - & - & - & - & - \\
\hline
\end{tabular}

Table 9. The $\tilde{f}_{11}(i, j)$ values obtained by our proposed algorithm.

\begin{tabular}{cccccccc}
\hline $\boldsymbol{i} / \boldsymbol{j}$ & $\mathbf{1}$ & $\mathbf{2}$ & $\mathbf{3}$ & $\mathbf{4}$ & $\mathbf{5}$ & $\mathbf{6}$ & $\mathbf{7}$ \\
\hline $\mathbf{1}$ & - & $(800,820,840)$ & $(350,361,370)$ & $(1000,1028,1253)$ & $(1080,1109,1140)$ & $(650,677,683)$ & $(410,430,500)$ \\
$\mathbf{2}$ & - & - & $(180,186,193)$ & $(830,853,1076)$ & $(495,510,525)$ & $(1105,1170,1215)$ & $(835,871,913)$ \\
$\mathbf{3}$ & - & - & - & $(650,667,883)$ & $(730,748,770)$ & $(960,1007,1243)$ & $(655,685,720)$ \\
$\mathbf{4}$ & - & - & - & - & $(190,199,210)$ & $(310,340,360)$ & - \\
$\mathbf{5}$ & - & - & - & - & - & $(610,660,690)$ & - \\
$\mathbf{6}$ & - & - & - & - & - & $(390,410,440)$ & - \\
$\mathbf{7}$ & - & - & - & - & - & $(620,652,695)$ & $(230,242,255)$ \\
$\mathbf{8}$ & - & - & - & $(710,730,735)$ & $(900,929,945)$ & $(510,540,590)$ & $(120,130,150)$ \\
$\mathbf{9}$ & - & - & - & $(840,867,880)$ & $(1030,1066,1090)$ & $(510)$ \\
$\mathbf{1 0}$ & - & - & - & - & - & $(720,752,790)$ & $(330,342,350)$ \\
$\mathbf{1 1}$ & - & - & - & - & - & - & - \\
\hline
\end{tabular}

Table 9. continued.

\begin{tabular}{ccccc}
\hline $\boldsymbol{i} / \boldsymbol{j}$ & $\mathbf{8}$ & $\mathbf{9}$ & $\mathbf{1 0}$ & $\mathbf{1 1}$ \\
\hline $\mathbf{1}$ & $(420,437,495)$ & $(290,300,350)$ & $(420,450,470)$ & $(840,882,990)$ \\
$\mathbf{2}$ & $(605,629,658)$ & $(900,930,960)$ & $(1130,1172,1220)$ & $(1265,1323,1403)$ \\
$\mathbf{3}$ & $(425,443,465)$ & - & - & $(1085,1137,1210)$ \\
$\mathbf{4}$ & - & - & - & $(540,582,620)$ \\
$\mathbf{5}$ & - & - & - & $(840,902,950)$ \\
$\mathbf{6}$ & - & - & - & $(230,242,260)$ \\
$\mathbf{7}$ & - & - & - & $(430,452,490)$ \\
$\mathbf{8}$ & - & - & $(230,242,260)$ & $(660,694,745)$ \\
$\mathbf{9}$ & $(130,137,145)$ & - & - & $(750,582,640)$ \\
$\mathbf{1 0}$ & - & - & - & - \\
$\mathbf{1 1}$ & - & - & & $(760,794,840)$ \\
\hline
\end{tabular}

Table 10. The $P_{11}(i, j)$ values obtained by our proposed algorithm.

\begin{tabular}{cccccccccccc}
\hline $\boldsymbol{i} / \boldsymbol{j}$ & $\mathbf{1}$ & $\mathbf{2}$ & $\mathbf{3}$ & $\mathbf{4}$ & $\mathbf{5}$ & $\mathbf{6}$ & $\mathbf{7}$ & $\mathbf{8}$ & $\mathbf{9}$ & $\mathbf{1 0}$ & $\mathbf{1 1}$ \\
\hline $\mathbf{1}$ & - & 1 & 1 & 3 & 3 & 1 & 9 & 9 & 1 & 1 & 9 \\
$\mathbf{2}$ & - & - & 2 & 3 & 2 & 5 & 8 & 3 & 2 & 9 & 8 \\
$\mathbf{3}$ & - & - & - & 3 & 3 & 4 & 8 & 3 & - & - & 8 \\
$\mathbf{4}$ & - & - & - & - & 4 & 4 & - & - & - & - & 6 \\
$\mathbf{5}$ & - & - & - & - & - & 5 & - & - & - & - & 6 \\
$\mathbf{6}$ & - & - & - & - & - & - & - & - & - & - & 6 \\
$\mathbf{7}$ & - & - & - & - & - & 7 & - & - & - & - & 7 \\
$\mathbf{8}$ & - & - & - & 8 & 4 & 7 & 8 & - & - & - & 7 \\
$\mathbf{9}$ & - & - & - & 8 & 8 & 7 & 9 & 9 & - & 9 & 7 \\
$\mathbf{1 0}$ & - & - & - & - & - & 7 & 10 & - & - & - & 7 \\
$\mathbf{1 1}$ & - & - & - & - & - & - & - & - & - & - & - \\
\hline
\end{tabular}


Table 11. The arc lengths for Example 3.

\begin{tabular}{cccccc}
\hline Arc & lengths & Arc & lengths & Arc & lengths \\
\hline$(1,2)$ & $(12,13,15,17)$ & $(1,3)$ & $(9,11,13,15)$ & $(1,4)$ & $(8,10,12,13)$ \\
$(1,5)$ & $(7,8,9,10)$ & $(2,6)$ & $(5,10,15,16)$ & $(2,7)$ & $(6,11,11,13)$ \\
$(3,8)$ & $(10,11,16,17)$ & $(4,7)$ & $(17,20,22,24)$ & $(4,11)$ & $(6,10,13,14)$ \\
$(5,8)$ & $(6,9,11,13)$ & $(5,11)$ & $(7,10,13,14)$ & $(5,12)$ & $(10,13,15,17)$ \\
$(6,9)$ & $(6,8,10,11)$ & $(6,10)$ & $(10,11,14,15)$ & $(7,10)$ & $(9,10,12,13)$ \\
$(7,11)$ & $(6,7,8,9)$ & $(8,12)$ & $(5,8,9,10)$ & $(8,13)$ & $(3,5,8,10)$ \\
$(9,16)$ & $(6,7,9,10)$ & $(10,16)$ & $(12,13,16,17)$ & $(10,17)$ & $(15,19,20,21)$ \\
$(11,14)$ & $(8,9,11,13)$ & $(11,17)$ & $(6,9,11,13)$ & $(12,14)$ & $(13,14,16,18)$ \\
$(12,15)$ & $(12,14,15,16)$ & $(13,15)$ & $(10,12,14,15)$ & $(13,19)$ & $(17,18,19,20)$ \\
$(14,21)$ & $(11,12,13,14)$ & $(15,18)$ & $(8,9,11,13)$ & $(15,19)$ & $(5,7,10,12)$ \\
$(16,20)$ & $(9,12,14,16)$ & $(17,20)$ & $(7,10,11,12)$ & $(17,21)$ & $(6,7,8,10)$ \\
$(18,21)$ & $(15,17,18,19)$ & $(18,22)$ & $(3,5,7,9)$ & $(18,23)$ & $(5,7,9,11)$ \\
$(19,22)$ & $(15,16,17,19)$ & $(20,23)$ & $(13,14,16,17)$ & $(21,23)$ & $(12,15,17,18)$ \\
$(22,23)$ & $(4,5,6,8)$ & & & & \\
\hline
\end{tabular}

Table 12. The $\tilde{f}_{23}(i, j)$ values obtained by our algorithm.

\begin{tabular}{|c|c|c|c|c|c|c|c|c|}
\hline$i / j$ & 1 & 2 & 3 & 4 & 5 & 6 & 7 & 8 \\
\hline 1 & - & $(12,13,15,17)$ & $(9,11,13,15)$ & $(8,10,12,13)$ & $(7,8,9,10)$ & $(17,23,30,33)$ & $(18,24,26,30)$ & $(13,17,20,23)$ \\
\hline 2 & - & - & - & - & - & $(5,10,15,16)$ & $(6,11,11,13)$ & - \\
\hline $\mathbf{3}$ & - & - & - & - & - & - & - & $(10,11,16,17)$ \\
\hline 4 & - & - & - & - & - & - & $(17,20,22,24)$ & - \\
\hline 5 & - & - & - & - & - & - & - & $(6,9,11,13)$ \\
\hline 6 & - & - & - & - & - & - & - & - \\
\hline 7 & - & - & - & - & - & - & - & - \\
\hline 8 & - & - & - & - & - & - & - & - \\
\hline 9 & - & - & - & - & - & - & - & - \\
\hline 10 & - & - & - & - & - & - & - & - \\
\hline 11 & - & - & - & - & - & - & - & - \\
\hline 12 & - & - & - & - & - & - & - & - \\
\hline 13 & - & - & - & - & - & - & - & - \\
\hline 14 & - & - & - & - & - & - & - & - \\
\hline 15 & - & - & - & - & - & - & - & - \\
\hline 16 & - & - & - & - & - & - & - & - \\
\hline 17 & - & - & - & - & - & - & - & - \\
\hline 18 & - & - & - & - & - & - & - & - \\
\hline 19 & - & - & - & - & - & - & - & - \\
\hline 20 & - & - & - & - & - & - & - & - \\
\hline 21 & - & - & - & - & - & - & - & - \\
\hline 22 & - & - & - & - & - & - & - & - \\
\hline 23 & - & - & - & - & - & - & - & - \\
\hline
\end{tabular}

Table 12. continued.

\begin{tabular}{|c|c|c|c|c|c|c|c|}
\hline$i / j$ & 9 & 10 & 11 & 12 & 13 & 14 & 15 \\
\hline 1 & $(23,31,40,44)$ & $(27,34,38,43)$ & $(14,18,22,24)$ & $(17,21,24,27)$ & $(16,22,28,33)$ & $(22,27,33,37)$ & $(29,35,39,43)$ \\
\hline 2 & $(11,18,25,27)$ & $(15,21,23,26)$ & $(12,18,19,22)$ & - & - & $(20,27,30,35)$ & - \\
\hline 3 & - & - & - & $(15,19,25,27)$ & $(13,16,24,27)$ & $(28,33,41,45)$ & $(23,28,38,42)$ \\
\hline 4 & - & $(26,30,34,37)$ & $(6,10,13,14)$ & - & - & $(14,19,24,27)$ & - \\
\hline 5 & - & - & $(7,10,13,14)$ & $(10,13,15,17)$ & $(9,14,19,23)$ & $(15,19,24,27)$ & $(22,27,30,33)$ \\
\hline 6 & $(6,8,10,11)$ & $(10,11,14,15)$ & - & - & - & - & - \\
\hline 7 & - & $(9,10,12,13)$ & $(6,7,8,9)$ & - & - & $(14,16,19,22)$ & - \\
\hline 8 & - & - & - & $(5,8,9,10)$ & $(3,5,8,10)$ & $(18,22,25,28)$ & $(13,17,22,25)$ \\
\hline 9 & - & - & - & - & - & - & - \\
\hline 10 & - & - & - & - & - & - & - \\
\hline 11 & - & - & - & - & - & $(8,9,11,13)$ & - \\
\hline 12 & - & - & - & - & - & $(13,14,16,18)$ & $(12,14,15,16)$ \\
\hline 13 & - & - & - & - & - & - & $(10,12,14,15)$ \\
\hline 14 & - & - & - & - & - & - & $(10,12,1,10)$ \\
\hline 15 & - & - & - & - & - & - & - \\
\hline 16 & - & - & - & - & - & - & - \\
\hline 17 & - & - & - & - & - & - & - \\
\hline 18 & - & - & - & - & - & - & - \\
\hline 19 & - & - & - & - & - & - & - \\
\hline 20 & - & - & - & - & - & - & - \\
\hline 21 & - & - & - & - & - & - & - \\
\hline 22 & - & - & - & - & - & - & - \\
\hline 23 & - & - & - & - & - & - & - \\
\hline
\end{tabular}


Table 12. continued.

\begin{tabular}{|c|c|c|c|c|c|c|c|c|}
\hline$i / j$ & 16 & 17 & 18 & 19 & 20 & 21 & 22 & 23 \\
\hline 1 & $(29,38,49,54)$ & $(20,27,33,37)$ & $(37,44,50,56)$ & $(33,40,47,53)$ & $(27,37,44,49)$ & $(26,34,41,47)$ & $(40,49,57,65)$ & $(38,49,58,65)$ \\
\hline 2 & $(17,25,34,37)$ & $(18,27,30,35)$ & - & - & $(25,37,41,47)$ & $(24,34,38,45)$ & - & $(36,49,55,63)$ \\
\hline 3 & - & - & $(31,37,49,55)$ & $(30,34,43,47)$ & - & $(39,45,54,59)$ & $(34,42,56,64)$ & $(36,44,58,66)$ \\
\hline 4 & $(38,43,50,54)$ & $(12,19,24,27)$ & - & - & $(19,29,35,39)$ & $(18,26,32,37)$ & - & $(30,41,49,55)$ \\
\hline 5 & - & $(13,19,24,27)$ & $(30,36,41,46)$ & $(26,32,38,43)$ & $(20,29,35,39)$ & $(19,26,32,37)$ & $(33,41,48,55)$ & $(31,41,49,55)$ \\
\hline 6 & $(12,15,19,21)$ & $(25,30,34,36)$ & - & - & $(21,27,33,37)$ & $(31,37,42,46)$ & - & $(34,41,49,54)$ \\
\hline 7 & $(21,23,28,30)$ & $(12,16,19,22)$ & - & - & $(19,26,30,34)$ & $(18,23,27,32)$ & - & $(30,38,44,50)$ \\
\hline 8 & - & - & $(21,26,33,38)$ & $(20,23,27,30)$ & - & $(29,34,38,42)$ & $(24,31,40,47)$ & $(26,33,42,49)$ \\
\hline 9 & $(6,7,9,10)$ & - & - & - & $(15,19,23,26)$ & - & - & $(28,33,39,43)$ \\
\hline 10 & $(12,13,16,17)$ & $(15,19,20,21)$ & - & - & $(21,25,30,33)$ & $(21,26,28,31)$ & - & $(34,39,46,50)$ \\
\hline 11 & - & $(6,9,11,13)$ & - & - & $(13,19,22,25)$ & $(12,16,19,23)$ & - & $(24,31,36,41)$ \\
\hline 12 & - & - & $(20,23,26,29)$ & $(17,21,25,28)$ & - & $(24,26,29,32)$ & $(23,28,33,38)$ & $(25,30,35,40)$ \\
\hline 13 & - & - & $(18,21,25,28)$ & $(17,18,19,20)$ & - & $(33,38,43,47)$ & $(21,26,32,37)$ & $(23,28,34,39)$ \\
\hline 14 & - & - & - & - & - & $(11,12,13,14)$ & - & $(23,27,30,32)$ \\
\hline 15 & - & - & $(8,9,11,13)$ & $(5,7,10,12)$ & - & $(23,26,29,32)$ & $(11,14,18,22)$ & $(13,16,20,24)$ \\
\hline 16 & - & - & - & - & $(9,12,14,16)$ & - & - & $(22,26,30,33)$ \\
\hline 17 & - & - & - & - & $(7,10,11,12)$ & $(6,7,8,10)$ & - & $(18,22,25,28)$ \\
\hline 18 & - & - & - & - & - & $(15,17,18,19)$ & $(3,5,7,9)$ & $(5,7,9,11)$ \\
\hline 19 & - & - & - & - & - & - & $(15,16,17,19)$ & $(19,21,23,27)$ \\
\hline 20 & - & - & - & - & - & - & - & $(13,14,16,17)$ \\
\hline 21 & - & - & - & - & - & - & - & $(12,15,17,18)$ \\
\hline 22 & - & - & - & - & - & - & - & $(4,5,6,8)$ \\
\hline 23 & - & - & - & - & - & - & - & - \\
\hline
\end{tabular}

Table 13. The $P_{23}(i, j)$ values obtained by our algorithm.

\begin{tabular}{|c|c|c|c|c|c|c|c|c|c|c|c|c|c|c|c|c|c|c|c|c|c|c|c|}
\hline$i / j$ & 1 & 2 & 3 & 4 & 5 & 6 & 7 & 8 & 9 & 10 & 11 & 12 & 13 & 14 & 15 & 16 & 17 & 18 & 19 & 20 & 21 & 22 & 23 \\
\hline 1 & - & 1 & 1 & 1 & 1 & 2 & 2 & 5 & 6 & 7 & 5 & 5 & 8 & 11 & 12 & 9 & 11 & 15 & 13 & 17 & 17 & 18 & 21 \\
\hline 2 & - & - & - & - & - & 2 & 2 & - & 6 & 7 & 7 & - & - & 11 & - & 9 & 11 & - & - & 17 & 17 & - & 21 \\
\hline 3 & - & - & - & - & - & - & - & 3 & - & - & - & 8 & 8 & 12 & 13 & - & - & 15 & 13 & - & 14 & 18 & 18 \\
\hline 4 & - & - & - & - & - & - & 4 & - & - & 7 & 4 & - & - & 11 & - & 10 & 11 & - & - & 17 & 17 & - & 21 \\
\hline 5 & - & - & - & - & - & - & - & 5 & - & - & 5 & 5 & 8 & 11 & 12 & - & 11 & 15 & 13 & 17 & 17 & 18 & 21 \\
\hline 6 & - & - & - & - & - & - & - & - & 6 & 6 & - & - & - & - & - & 9 & 10 & - & - & 16 & 17 & - & 20 \\
\hline 7 & - & - & - & - & - & - & - & - & - & 7 & 7 & - & - & 11 & - & 10 & 11 & - & - & 17 & 17 & - & 21 \\
\hline 8 & - & - & - & - & - & - & - & - & - & - & - & 8 & 8 & 12 & 13 & - & - & 15 & 13 & - & 14 & 18 & 18 \\
\hline 9 & - & - & - & - & - & - & - & - & - & - & - & - & - & - & - & 9 & - & - & - & 16 & - & - & 20 \\
\hline 10 & - & - & - & - & - & - & - & - & - & - & - & - & - & - & - & 10 & 10 & - & - & 16 & 17 & - & 20 \\
\hline 11 & - & - & - & - & - & - & - & - & - & - & - & - & - & 11 & - & - & 11 & - & - & 17 & 17 & - & 21 \\
\hline 12 & - & - & - & - & - & - & - & - & - & - & - & - & - & 12 & 12 & - & - & 15 & 15 & - & 14 & 18 & 18 \\
\hline 13 & - & - & - & - & - & - & - & - & - & - & - & - & - & - & 13 & - & - & 15 & 13 & - & 18 & 18 & 18 \\
\hline 14 & - & - & - & - & - & - & - & - & - & - & - & - & - & - & - & - & - & - & - & - & 14 & - & 21 \\
\hline 15 & - & - & - & - & - & - & - & - & - & - & - & - & - & - & - & - & - & 15 & 15 & - & 18 & 18 & 18 \\
\hline 16 & - & - & - & - & - & - & - & - & - & - & - & - & - & - & - & - & - & - & - & 16 & - & - & 20 \\
\hline 17 & - & - & - & - & - & - & - & - & - & - & - & - & - & - & - & - & - & - & - & 17 & 17 & - & 21 \\
\hline 18 & - & - & - & - & - & - & - & - & - & - & - & - & - & - & - & - & - & - & - & - & 18 & 18 & 18 \\
\hline 19 & - & - & - & - & - & - & - & - & - & - & - & - & - & - & - & - & - & - & - & - & - & 19 & 22 \\
\hline 20 & - & - & - & - & - & - & - & - & - & - & - & - & - & - & - & - & - & - & - & - & - & - & 20 \\
\hline 21 & - & - & - & - & - & - & - & - & - & - & - & - & - & - & - & - & - & - & - & - & - & - & 21 \\
\hline 22 & - & - & - & - & - & - & - & - & - & - & - & - & - & - & - & - & - & - & - & - & - & - & 22 \\
\hline 23 & - & - & - & - & - & - & - & - & - & - & - & - & - & - & - & - & - & - & - & - & - & - & - \\
\hline
\end{tabular}




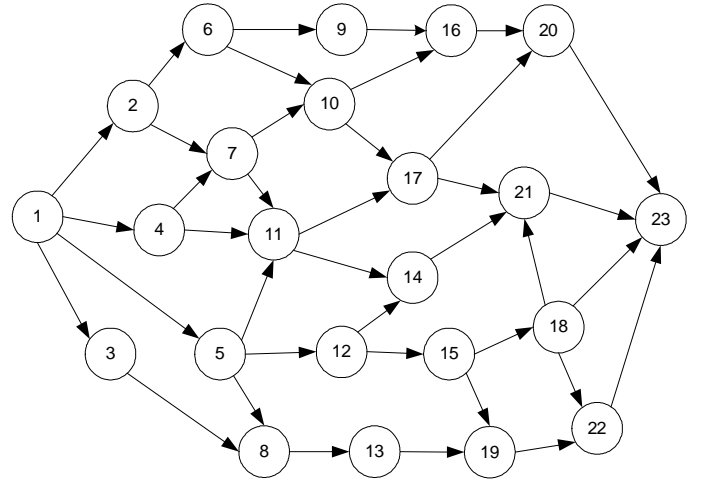

Figure 6. The telecommunication network proposed for Example 3.

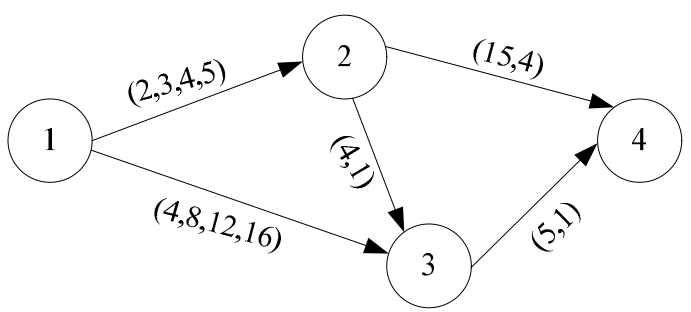

Figure 7. A small sized network having various fuzzy arc lengths.

Table 14. The $\tilde{f}_{0}(i, j)$ matrix for $\boldsymbol{k}=\mathbf{0}$.

\begin{tabular}{ccccc}
\hline $\boldsymbol{i} / \boldsymbol{j}$ & $\mathbf{1}$ & $\mathbf{2}$ & $\mathbf{3}$ & $\mathbf{4}$ \\
\hline $\mathbf{1}$ & - & $(2,3,4,5)$ & $(4,8,12,16)$ & 2 \\
$\mathbf{2}$ & - & - & $(4,1)$ & $(15,4)$ \\
$\mathbf{3}$ & - & - & - & $(5,1)$ \\
$\mathbf{4}$ & - & - & - & - \\
\hline
\end{tabular}

Therefore, with $P_{k}(i, j)=i$, we have Table 15 .

Step 2: Here, we consider $k=1$ and compute the value of $f_{k}(i, j)=\min \left[f_{k-1}(i, j), f_{k-1}(i, k)+f_{k-1}(k, j)\right]$. The result is shown in Table 16.

Therefore, for $P_{k}(i, j)=i$, we have Table 17 .

Table 15. The $P_{0}(i, j)$ matrix for $\boldsymbol{k}=\mathbf{0}$.

\begin{tabular}{lllll}
\hline $\boldsymbol{i} / \boldsymbol{j}$ & $\mathbf{1}$ & $\mathbf{2}$ & $\mathbf{3}$ & $\mathbf{4}$ \\
\hline $\mathbf{1}$ & - & 1 & 1 & - \\
$\mathbf{2}$ & - & - & 2 & 2 \\
$\mathbf{3}$ & - & - & - & 3 \\
$\mathbf{4}$ & - & - & - & -
\end{tabular}

Table 16. The $\tilde{f}_{1}(i, j)$ matrix for $k=1$.

\begin{tabular}{lllll}
\hline $\boldsymbol{i} / \boldsymbol{j}$ & $\mathbf{1}$ & $\mathbf{2}$ & $\mathbf{3}$ & $\mathbf{4}$ \\
\hline $\mathbf{1}$ & - & $(2,3,4,5)$ & $(4,8,12,16)$ & - \\
$\mathbf{2}$ & - & - & $(4,1)$ & $(15,4)$ \\
$\mathbf{3}$ & - & - & - & $(5,1)$ \\
$\mathbf{4}$ & - & - & - & - \\
\hline
\end{tabular}

Table 17. The $P_{1}(i, j)$ matrix for $k=1$.

\begin{tabular}{lllll}
\hline $\boldsymbol{i} / \boldsymbol{i}$ & $\mathbf{1}$ & $\mathbf{2}$ & $\mathbf{3}$ & $\mathbf{4}$ \\
\hline $\mathbf{1}$ & - & 1 & 1 & - \\
$\mathbf{2}$ & - & - & 2 & 2 \\
$\mathbf{3}$ & - & - & - & 3 \\
$\mathbf{4}$ & - & - & - & - \\
\hline
\end{tabular}

If node $k$ is not on the shortest path using $\{1,2, \ldots, k\}$ as intermediate nodes, then we consider $P_{k}(i, j)=P_{k-1}(i, j)$, otherwise we let $P_{k}(i, j)=P_{k-1}(i, k)$. We now report the results obtained for other values of $k$ in Tables 18-23. Note that, the sets $V_{i}$ and $W_{i}$ are the points obtained by $\alpha$-cut additions, where the $V$ and $W$ values are obtained by the $\alpha_{i}$-cuts considering $n=10$. It includes 10 points for the $a_{\alpha_{i}}^{-}$and 10 points for the $a_{\alpha_{i}}^{+}$:

$$
\begin{aligned}
\mathrm{V}_{1}=\{ & (4.58257,10.4174),(4.93136,10.0686), \\
& (5.20274,9.79726),(5.44277,9.55723), \\
& (5.66745,9.33255),(5.88528,9.11472), \\
& (6.10278,8.89722),(6.32762,8.67238), \\
& (6.57541,8.42459),(7,8)\}
\end{aligned}
$$

$\mathrm{W}_{1}=\{(11.0303,25.9697),(12.1255,24.8745)$, (12.911,24.089), (13.5711,23.4289), (14.1698,22.8302), (14.7411,22.2589), $(15.3111,21.6889),(15.9105,21.0895)$, $(16.6016,20.3984),(18,19)\}$

$V_{2}=\{(4.58257,10.4174),(4.93136,10.0686)$, (5.20274,9.79726), (5.44277,9.55723), $(5.66745,9.33255),(5.88528,9.11472)$, $(6.10278,8.89722),(6.32762,8.67238)$, $(6.57541,8.42459),(7,8)\}$

$\mathrm{W}_{2}=\{(8.06515,16.9349),(8.66273,16.3373)$, $(9.10549,15.8945),(9.48554,15.5145)$, $(9.83489,15.1651),(10.1706,14.8294)$, (10.5056,14.4944), (10.8552,14.1448), $(11.2508,13.7492),(12,13)\}$

Table 18. The $\tilde{f}_{2}(i, j)$ matrix for $k=2$.

\begin{tabular}{lllll}
\hline $\boldsymbol{i} / \boldsymbol{j}$ & $\mathbf{1}$ & $\mathbf{2}$ & $\mathbf{3}$ & $\mathbf{4}$ \\
\hline $\mathbf{1}$ & - & $(2,3,4,5)$ & $\mathrm{V}_{1}$ & $\mathrm{~W}_{1}$ \\
$\mathbf{2}$ & - & - & $(4,1)$ & $(15,4)$ \\
$\mathbf{3}$ & - & - & - & $(5,1)$ \\
$\mathbf{4}$ & - & - & - & - \\
\hline
\end{tabular}

Table 19. The $P_{2}(i, j)$ matrix for $k=2$.

\begin{tabular}{ccccc}
\hline $\boldsymbol{i} / \boldsymbol{j}$ & $\mathbf{1}$ & $\mathbf{2}$ & $\mathbf{3}$ & $\mathbf{4}$ \\
\hline $\mathbf{1}$ & - & 1 & 2 & 2 \\
$\mathbf{2}$ & - & - & 2 & 2 \\
$\mathbf{3}$ & - & - & - & 3 \\
$\mathbf{4}$ & - & - & - & - \\
\hline
\end{tabular}

Table 20. The $\tilde{f}_{3}(i, j)$ matrix for $k=3$

\begin{tabular}{lllll}
\hline $\boldsymbol{i} / \boldsymbol{j}$ & $\mathbf{1}$ & $\mathbf{2}$ & $\mathbf{3}$ & $\mathbf{4}$ \\
\hline $\mathbf{1}$ & - & $(2,3,4,5)$ & $\mathrm{V}_{2}$ & $\mathrm{~W}_{2}$ \\
$\mathbf{2}$ & - & - & $(4,1)$ & $(9,2)$ \\
$\mathbf{3}$ & - & - & - & $(5,1)$ \\
$\mathbf{4}$ & - & - & - & - \\
\hline
\end{tabular}

Table 21. The $P_{3}(i, j)$ matrix for $k=3$.

\begin{tabular}{lllll}
\hline $\boldsymbol{i} / \boldsymbol{j}$ & $\mathbf{1}$ & $\mathbf{2}$ & $\mathbf{3}$ & $\mathbf{4}$ \\
\hline $\mathbf{1}$ & - & 1 & 2 & 3 \\
$\mathbf{2}$ & - & - & 2 & 3 \\
$\mathbf{3}$ & - & - & - & 3 \\
$\mathbf{4}$ & - & - & - & - \\
\hline
\end{tabular}


Table 22. The $\tilde{f}_{4}(i, j)$ matrix for $k=4$.

\begin{tabular}{lllll}
\hline $\boldsymbol{i} / \boldsymbol{j}$ & $\mathbf{1}$ & $\mathbf{2}$ & $\mathbf{3}$ & $\mathbf{4}$ \\
\hline $\mathbf{1}$ & - & $(2,3,4,5)$ & $\mathrm{V}_{3}$ & $\mathrm{~W}_{3}$ \\
$\mathbf{2}$ & - & - & $(4,1)$ & $(9,2)$ \\
$\mathbf{3}$ & - & - & - & $(5,1)$ \\
$\mathbf{4}$ & - & - & - & - \\
\hline
\end{tabular}

Table 23. The $P_{4}(i, j)$ matrix for $k=4$.

\begin{tabular}{lllll}
\hline $\boldsymbol{i} / \boldsymbol{j}$ & $\mathbf{1}$ & $\mathbf{2}$ & $\mathbf{3}$ & $\mathbf{4}$ \\
\hline $\mathbf{1}$ & - & 1 & 2 & 3 \\
$\mathbf{2}$ & - & - & 2 & 3 \\
$\mathbf{3}$ & - & - & - & 3 \\
$\mathbf{4}$ & - & - & - & - \\
\hline
\end{tabular}

$\mathrm{V}_{3}=\{(4.58257,10.4174),(4.93136,10.0686)$, (5.20274,9.79726), (5.44277,9.55723), (5.66745,9.33255), (5.88528,9.11472), (6.10278,8.89722), (6.32762,8.67238), $(6.57541,8.42459),(7,8)\}$

$\mathrm{W}_{3}=\{(8.06515,16.9349),(8.66273,16.3373)$, (9.10549,15.8945), (9.48554,15.5145), (9.83489,15.1651), (10.1706,14.8294), (10.5056,14.4944), (10.8552,14.1448), $(11.2508,13.7492),(12,13)\}$

Finally, when $k=N$, we identify the shortest path as follows:

Shortest path from 1 to $4: 1-2-3-4$.

Shortest path length from 1 to 4 :

$(8.06515,16.9349),(8.66273,16.3373),(9.10549,15.894$

5),(9.48554,15.5145),(9.83489,15.1651),(10.1706,14.829

4),(10.5056,14.4944),(10.8552,14.1448),(11.2508,13.749 $2),(12,13)$

\section{Conclusions}

We presented a novel approach for computing a shortest path in a mixed network having various fuzzy arc lengths. First, we developed a new technique for the addition of various fuzzy numbers in a path using $\alpha$-cuts. Then, we applied a dynamic programming method for finding a shortest path in the network, using a recently proposed distance function to compare fuzzy numbers in the proposed algorithm. Four comparative examples were worked out to illustrate the applicability of our proposed approach as compared to two other methods in the literature as well as demonstrate the additional novel feature offered by our algorithm to find a fuzzy shortest path in mixed fuzzy networks having various settings for the fuzzy arc lengths.

\section{References}

[1] R. E. Moore, "Method and application of interval analysis," SIAM, Philadelphia, 1997. M. Delgado, J. L. Ver- degay, and M. A. Vila, "A procedure for ranking fuzzy numbers using fuzzy relations," Fuzzy Sets and Systems, Vol. 26, pp. 49-62, 1988.

[2] M. Delgado, J. L. Verdegay, and M. A. Vila, "A procedure for ranking fuzzy numbers using fuzzy relations," Fuzzy Sets and Systems, Vol. 26, pp. 49-62, 1988.

[3] H. Ishibuchi and H. Tanaka, "Multi-objective programming in optimization of the interval objective function," European Journal of Operational Research, Vol. 48, pp. 219-225, 1990.

[4] J. K. Sengupta, "Optimal decision under uncertainty," Springer, New York, 1981.

[5] T. Shaocheng, "Interval number and fuzzy number linear programming," Fuzzy Sets and Systems, Vol. 66, pp. 301-306. 1994.

[6] A. Sengupta, T. K. Pal, "Theory and methodology on comparing interval numbers," European Journal of Operational Research, Vol. 127, 28-43, 2000.

[7] D. Dubois and H. Prade, "Ranking fuzy numbers in the setting of possibility theory," Information Sciences, Vol. 30, pp. 183-224, 1983.

[8] S. H. Chen, "Ranking fuzzy numbers with maximizing set and minimizing set," Fuzzy Sets and Systems, Vol. 17, pp. 113-129, 1985.

[9] G. Bortolan and R. Degani, "A review of some methods for ranking fuzzy subsets," Fuzzy Sets and Systems, Vol. 15, pp. 1-19, 1985.

[10] C.-H. Cheng, "A new approach for ranking fuzzy numbers by distance method," Fuzzy Sets and Systems, Vol. 95, pp. 307-317, 1998.

[11] M. Blue, B. Bush, and J. Puckett, "Unified approach to fuzzy graph problems," Fuzzy Sets and Systems, Vol. 125, pp. 355-368, 2002.

[12] L. T. Koczy, "Fuzzy graphs in the evaluation and optimization of networks," Fuzzy Sets and Systems, Vol. 46, pp. 307-319, 1992.

[13] C. M. Klein, "Fuzzy Shortest Paths," Fuzzy Sets and Systems, Vol. 39, pp. 27-41, 1991.

[14] Y. Li, M. Gen, and K. Ida, "Solving fuzzy shortest path problem by neural networks," Computers Industrial Engineering, Vol. 31, pp. 861-865, 1996.

[15] K. Lin and M. Chen, "The fuzzy shortest path problem and its most vital arcs," Fuzzy Sets and Systems, Vol. 58, pp. 343-353, 1994.

[16] S. Okada and M. Gen, "Order relation between intervals and its application to shortest path problem," Computers \& Industrial Engineering, Vol. 25, 147-150, 1993.

[17] S. Okada and M. Gen, "Fuzzy shortest path problem," Computers \& Industrial Engineering, Vol. 27, pp. 465468, 1994.

[18] S. Okada and T. Soper, "A shortest path problem on a network with fuzzy arc lengths," Fuzzy Sets and Systems, Vol. 109, pp. 129-140, 2000.

[19] L. A. Zadeh, "Fuzzy sets as a basis for a theory of possibility," Fuzzy Sets and Systems, Vol. 1, pp. 3-28, 1978.

[20] R. K. Ahuja, K. Mehlhorn, J. B. Orlin, and R. E. Tarjan, 
"Faster algorithms for the shortest path problem," Technical Report CS-TR-154-88, Department of Computer Science, Princeton University, 1988.

[21] A. Andersson, T. Hagerup, S. Nilsson, and R. Raman, "Sorting in linear time?" In Proceedings of 27th ACM Symposium on Theory of Computing, pp. 427-436, 1995.

[22] A. V. Goldberg, "Scaling algorithms for the shortest paths problem," In: Proceedings of the 4th ACM-SIAM Symposium on Discrete Algorithms, pp. 222-231, 1993.

[23] Y. Asano and H. Imai, "Practical efficiency of the linear-time algorithm for the single source shortest path problem," Journal of the Operations Research Society of Japan, Vol. 43, pp. 431-447, 2000.

[24] Y. Han, "Improved algorithm for all pairs shortest paths," Information Processing Letters, Vol. 91, pp. 245-250, 2004.

[25] S. Saunders, T. Takaoka, "Improved shortest path algorithms for nearly acyclic graphs," Electronic Notes in Theoretical Computer Science, Vol. 42, pp. 1-17, 2001.

[26] S. Chanas, "Fuzzy optimization in networks," In: J. Kacprzyk, S. A. Orlovski (Eds.), Optimization Models Using Fuzzy Sets and Possibility Theory, D. Reidel, Dordrecht, pp. 303-327, 1987.
[27] B. Sadeghpour-Gildeh, D. Gien, Q. La Distance-Dp, et al. "Cofficient de Corrélation entre deux Variables Aléatoires floues," Actes de LFA'01, pp. 97-102, MonseBelgium, 2001.

[28] I. Mahdavi, R. Nourifar, A. Heidarzade, and N. MahdaviAmiri, "A dynamic programming approach for finding shortest chains in a fuzzy network," Applied Soft Computing, Vol. 9, No. 2, pp. 503-511, 2009.

[29] R. W. Floyd, Algorithm 97, Shortest path, CACM 5, pp. $345,1962$.

[30] T.-N. Chuang, and J.-Y. Kung, The fuzzy shortest path length and the corresponding shortest path in a network, Computers \& Operations Research, Vol. 32, 1409-1428, 2005.

[31] M. L. Fredman and R. E. Tarjan, "Fibonacci heaps and their uses in improved network optimization algorithm," J. ACM 34, Vol. 3, pp. 596-615, 1987.

[32] F. Hernandes, M. T. Lamata, J. L. Verdegay, and A. Yamakami, "The shortest path problem on networks with fuzzy parameters," Fuzzy Sets and Systems, Vol. 158, pp. 1561-1570, 2007. 